\title{
Perspektiven der Witzforschung im Kontext des Russischunterrichts
}

\author{
Julia Hargaßner (Salzburg)
}

Статья рассматривает немецкоязычное понятие «Witz», переводимое на русский язык как ,шутка', ,анекдот', ,острота', в контексте преподавания русского как иностранного (РКИ). Несмотря на то, что шутки и анекдоты на иностранном языке, а точнее их понимание и активное использование, выступают признаком компетентного в^адения языком, применение этого материала на уроках ограничено в силу его многогранности и сложности этой повествовательной формы. Автор статьи представляет перспективы изучения шутки как текста и речевого жанра в контексте преподавания РКИ, раскрывая ее потенциал. Кроме того, в статье приводятся результаты пилотного проекта, проведенного на отАелении славистики университета Зальцбурга. При помощи анкетирования выявлялось субъективное отношение студентов русского языка к использованию шуток на уроках, а именно, их влияние на атмосферу в классе, частотность использования шутки на уроках РКИ, а также связь между отношением к шутке на родном и иностранном языках.

\section{1. $\quad$ Einleitung}

Als Einstieg soll folgender russischer Witz dienen: „Поймац старик золотую рыбку и загадывает желание: - Хочу, чтобы у меня все было. Рыбка ему в ответ: - Старик, у тебя все было... “ Um über diesen Witz lachen oder wenigstens schmunzeln zu können, muss man sowohl die Bedeutung der Wörter als auch die grammatische Verbindung zwischen ihnen verstehen und selbstverständlich den kulturellen Kontext kennen. Es geht um das Gedicht „Skazka o rybake i rybke“ von Aleksandr Puškin, in dem ein alter armer Fischer einen Gold-

Anka Bergmann, Olga Caspers \& Wolfgang Stadler (Hg.)

Didaktik der slawischen Sprachen - Beiträge zum 1. Arbeitskreis in Berlin (12.-14.9.2016)

(C) 2018 innsbruck university press, ISBN 978-3-903187-11-5, DOI 10.15203/3187-11-5 
fisch fängt, der drei Wünsche des alten Mannes erfüllt, um als Gegenleistung frei gelassen zu werden. Die Polysemie des Wortes bylo (der Konjunktiv im Wunsch des Fischers und das Präteritum in der Antwort des Goldfischs) lässt sich in zwei unterschiedlichen Skripten lesen, die zur Pointe beitragen, in der eine überraschende Umdeutung stattfindet: Während der alte Mann sich ein sorgloses Leben wünscht, sagt ihm der Fisch, dass es schon der Vergangenheit angehört. Aus diesem kleinen Beispiel wird deutlich, dass der Witz zum einen mehrere Ebenen der Sprache (lexikalische, semantische, grammatische, phonologische, kulturelle) aktualisiert und zum anderen nur unter Verlust des komischen Effekts aus dem Russischen ins Deutsche übertragen werden kann.

Das Verstehen eines Witzes in einer fremden Sprache stellt mehrere Herausforderungen dar, zugleich aber vermittelt es das befriedigende Gefühl der kompetenten Beherrschung dieser Sprache. Welche Voraussetzungen gibt es für das Verstehen von Witzen in der Fremdsprache? Welche Kenntnisse sowohl auf der sprachlichen als auch der kulturellen Ebene benötigt der Lernende dazu? Welche Haltung haben UnterrichtsteilnehmerInnen zu Witzen im Fremdsprachenunterricht?

Für den Einsatz von Witzen bei der Vermittlung einer fremden Sprache sprechen v. a. die emotionale Komponente, die die Stimmung heben soll, und der relativ kurze Umfang der Texte, die sich im Rahmen einer Unterrichtsstunde sehr gut aufbereiten lassen. Demgegenüber stehen einige Aspekte, die den Einsatz von Witzen im Fremdsprachenunterricht erschweren, wie bspw. die hohen Sprachkompetenzniveaustufen im GeR (2001), auf denen die sowohl allgemeinen als auch sprachlich-kommunikativen und interkulturellen Kompetenzen der Lernenden in Verbindung mit Witzen gebracht werden. So sollen die Lernenden im Rahmen der Diskurskompetenz, die in den pragmatischen Kompetenzen verortet wird, wissen, „wie Geschichten, Anekdoten, Witze etc. erzählt werden“ (ebd.: 5.2.3.1). Im Kapitel soziolinguistische Kompetenz des GeR wird deutlich auf die Kenntnis des akkumulierten Volkswissens, ausgedrückt in Redewendungen als eine bedeutsame Komponente des sprachlichen Aspekts der soziokulturellen Kompetenz hingewiesen (ebd.: 5.2.2.3). Die Textsorte „Witz“ wird dabei nicht explizit erwähnt, abgesehen von der Skala der soziolinguistischen Angemessenheit für das Niveau C1: „Kann die Sprache zu geselligen Zwecken flexibel und 
effektiv einsetzen und dabei Emotionen ausdrücken, Anspielungen und Scherze machen." (ebd.: 5.2.2.5).

Witze nehmen im Kontext des Russischunterrichts eine besondere Stellung ein: Sie kommen zum einen in der schriftlichen Sprache als eine Textsorte und zum anderen in der mündlichen Sprache als eine Redegattung vor. Diese Zweiteilung wird mit der Aktivierung unterschiedlicher Aktivitäten in der (unterrichtlichen) Kommunikation begründet und folgt dem zeitgenössischen Paradigma der russischsprachigen Witzforschung (Chimik 2002, Dement'ev 2010, Kurganov 2015, Šmeleva \& Šmelev 2002). Witze beziehen sich auf alle Bereiche des menschlichen Lebens und repräsentieren die Kultur der jeweiligen Sprachgesellschaft. Die historische Entwicklung russischer Witze im 20. Jahrhundert spiegelt nicht nur politische, wirtschaftliche, geschlechterspezifische und soziale Veränderungen wider (vgl. Dement'ev 2010), sondern veranschaulicht auch wichtige Diskursparadigmen des Landes. So zeigen diachrone Untersuchungen russischer Witze eine Übereinstimmung zwischen den Phasen der gesellschaftlichen Entwicklung und der Witzentwicklung im Russland des 20. und 21. Jahrhunderts (ebd.). Diese Eigenschaften von Witzen sprechen für ihre Verwendung im unterrichtlichen Kontext.

Dabei bleibt die Frage offen, wann, wie, wie oft und ob überhaupt Witze als Textsorte und Redegattung ihren Einsatz im Russischunterricht finden. Einen möglichen Hinweis liefern Lehrwerke, die als „zentrales Medium des Fremdsprachenunterrichts" (Fäcke 2016: 35) betrachtet werden. Ohne einen Anspruch auf eine Analyse gegenwärtiger Lehrwerke hinsichtlich der Präsenz von Witzen zu stellen, führe ich ein Beispiel aus dem von mir im Unterricht verwendeten Lehrwerk Russkij klass (Vochmina \& Osipova 2011: 104) an. Hier werden auf einer Seite zwei Texte unter dem Titel „Witz mymкa“ angegeben (s. Abb. 1), die jedoch nur durch eine möglicherweise ähnliche Reaktion bei der Rezeption dem Bereich des Humors zugeordnet werden können.

Während der erste Witz eine pointierte Geschichte darstellt, weist der Reimwitz keine Anzeichen der Pointe auf. Das Beispiel verdeutlicht, dass der Witz (иутка) im Russischen als Sammelbegriff für unterschiedliche Textsorten agiert. An dieser Stelle soll angemerkt werden, dass das als Beispiel verwendete Lehrwerk ab der dritten Lektion in jedem Kapitel mind. einen Witz anführt, was einen systematischen Einsatz von Witzen im Unterricht nahelegen könnte.

Anka Bergmann, Olga Caspers \& Wolfgang Stadler (Hg.)

Didaktik der slawischen Sprachen - Beiträge zum 1. Arbeitskreis in Berlin (12.-14.9.2016)

(C) 2018 innsbruck university press, ISBN 978-3-903187-11-5, DOI 10.15203/3187-11-5 
27

Прочитайте и улыбнитесь.

Врач спра́шивает пацие́нта.

- Скажи́те, быва́ет так, что сль́шите го́лос, но не ви́дите, кто говори́т?

- Да, до́ктор, быва́ет.

- И ча́сто э́то быва́ет?

- Да, до́ктор, о́чень ча́сто.

- O, ә́то о́чень, о́чень пло́хо. А когда́ э́то быва́ет?

- Когда́ я говорю́ по телефо́ну.

28

Выучите наизусть стихотворение-шутку.

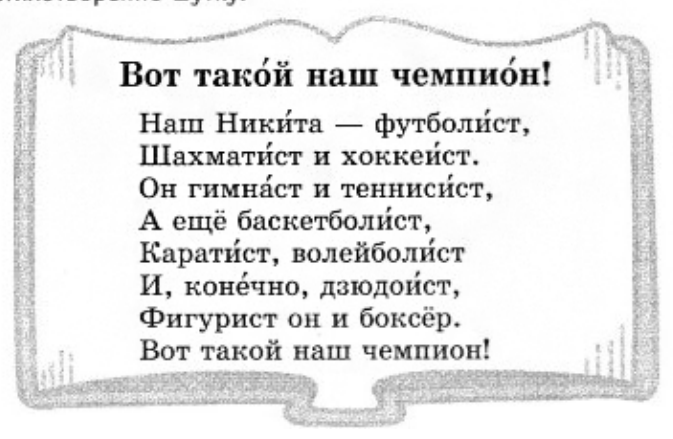

Abbildung 1: Seite aus dem Lehrwerk Russkij klass (Vochmina \& Osipova 2011: 104)

Abgesehen von Witzen in Lehrwerken sind in den letzten Jahren einige Lehrmittel erschienen, die sich explizit Witzen im Unterricht widmen (Lebedeva 2012, Levina 1997). Es handelt sich dabei um Sammlungen von Witzen, Anekdoten, Reimen und lustigen Dialogen, also humoresken Texten. Sie bestätigen wiederum das große Interesse an diesem Thema und die breitere Auffassung des (deutschen) Begriffs „Witz“ im Russischen.

Die einführenden Überlegungen zeigen, dass Witze ein vielseitiges Medium für den fremdsprachlichen Russischunterricht darstellen: Der Einsatz von Witzen im Russischunterricht ist aufgrund ihrer Mehrdimensionalität und Komplexität eine große Herausforderung im gesteuerten Spracherwerb. Genau dieselben Eigenschaften von Witzen sprechen aber für ihre Einbeziehung in den Sprachunterricht. Der folgende Beitrag hat zum Ziel, einen Forschungsüberblick in Bezug auf Witze darzubieten, die Definition des Witzes im Kontext des Russischunter- 
richts zu formulieren und eine neue Perspektive fachdidaktischer Witzforschung zu umreißen. Anschließend wird ein Pilotprojekt vorgestellt, das die Relevanz dieser Perspektive der Witzforschung verdeutlicht.

\section{Zur Definition des Witzes im Kontext des Russisch- unterrichts}

Wie bereits oben gezeigt, kommen in Lehrwerken abgesehen von den kurzen, eine Pointe enthaltenden Texten, die man als Witz bezeichnet, auch Texte vor, die mit Humor und Komik in Verbindung gebracht und verallgemeinernd auch als Witze angeführt werden. Somit besteht ein Klärungsbedarf hinsichtlich der Definition des Witzes im Russischunterricht. Dabei sollten sowohl die Definitionen aus den Bezugsdisziplinen als auch die Auslegung des Begriffs im russischsprachigen kulturellen Raum mit aufgenommen werden.

Unterschiedliche Herangehensweisen an das Wesen des Witzes fördern mehrere Definitionen zutage. Im historischen Verlauf kristallisierte sich die Auffassung des Witzes von geistiger Veranlagung zu sprachlichem Gebilde (vgl. Preisendanz 1970) heraus. Die Linguistik verwendet in Bezug auf den Witz folgende Begriffe: Textsorte, Handlungsmuster, Redegattung (Marfurt 1977, Raskin 2008). Kotthoff (2011: 397), eine der bekanntesten Humorforscherinnen der Gegenwart im deutschsprachigen Raum, gibt eine kurze Definition des Witzes: „Witze sind standardisierte Geschichten, die mit einer Pointe enden“. Ulrich (1980: 20) nennt den Witz eine „relativ kurze, komische und pointierte Sprach-, Bild- oder Bild-Sprach-Textsorte" und versucht den Witz von anderen Textsorten abzugrenzen. Bereits in den 1970er Jahren weist Marfurt (1977: 167) auf gewisse Probleme der Abgrenzung der Textsorte „Witz“ hin, mit der Begründung, dass „die Kriterien einer Textsortenbestimmung sowohl von der Textstruktur wie vom Interaktionsmuster her determiniert sind“. Die Problematik einer definitorischen Abgrenzung der Textsorte oder Redegattung "Witz“ ergibt sich aus der Vielfalt der Arten und Typen von Witzen sowie aus der essentiellen Ambiguität des Begriffs, der sich sowohl ,auf den Bereich des Verstandes [...] als auch auf

Anka Bergmann, Olga Caspers \& Wolfgang Stadler (Hg.)

Didaktik der slawischen Sprachen - Beiträge zum 1. Arbeitskreis in Berlin (12.-14.9.2016)

(C) 2018 innsbruck university press, ISBN 978-3-903187-11-5, DOI 10.15203/3187-11-5 
den Bereich des Komischen“" (Winkler \& Goulding 2005: 694-695), als Textsorte oder Gattung, bezieht. Mehrere aus unterschiedlichen Perspektiven gestaltete Definitionsversuche „verleihen der Textsorte kein deutlicheres Profil“ (ebd.: 696).

Die Definition der Textsorte "Witz“ im Rahmen des Russischunterrichts muss auch den heute üblichen Sprachgebrauch dieses Begriffs im russischsprachigen Raum miteinbeziehen. Zunächst bedarf es einer Übersetzung des deutschen Worts „Witz“, für das im Russischen gleich drei Bezeichnungen existieren: иутка, острота, und анекдоm (vgl. Lejn et al. 2006: 968). Das erklärende Wörterbuch der russischen Sprache definiert das Wort „иутка“ wie folgt: „1. То, что говорят, делают с целью вызвать смех, веселье; забавная, выходка, шалость. 2. То, что говорят и делают не всерьёз, к чему нельзя относиться серьёзно. 3. Небольшая комическая пьеса, комический рассказ, комическое музыкальное произведение“ (Efremova 2006: 899). Im Russischen sind die Grenzen zwischen den Textsorten „иутка“ und „анекдот“" oft verwischt. Es gibt eine große Anzahl an populärwissenschaftlichen Büchern, die in ihren Titeln diese Wörter synonym verwenden. Die wissenschaftliche Monographie Russkij anekdot: Tekst i rečevoj žanr (Šmeleva \& Šmelev 2002: 20) bietet die Definition des russischen Witzes als „Анекдот - короткий устный смешной рассказ о вымышленном событии с неожиАанной остроумной концовкой, в котором Аействуют постоянные персонажи, известные всем носителям русского языка“. Im Deutschen stellt der Witz ein Hyperonym dar, während sich die russische Bezeichnung анекдоm auf eine konkrete sprachlich-kulturelle Erscheinung bezieht (Chimik 2002). Winkler \& Goulding (2005) verweisen auch auf den Unterschied zwischen dem deutschen Nomen und „seinen Korrelaten in den anderen europäischen Sprachen: Diese verfügen über jeweils zwei Termini oder zwei Gruppen von Termini, um einerseits den Witz als geistige Anlage [...], andererseits den Witz als einzelnen Text zu bezeichnen“ (ebd.: 695). Unter „ocmpoma“ wird „остроумное выражение“, ein scharfsinniger Ausdruck, verstanden (Efremova 2006: 500). Obwohl es im Russischen drei Bezeichnungen für das gibt, was wir im Deutschen einen Witz nennen, wird die Definition und genaue Abgrenzung der damit bezeichneten Textsorte(n) dadurch nicht einfacher.

Im Rahmen der fremdsprachlichen Didaktik des Russischen scheint es sinnvoll zu sein, die oben dargelegten deutschsprachigen und russischsprachigen Aus-

Anka Bergmann, Olga Caspers \& Wolfgang Stadler (Hg.)

Didaktik der slawischen Sprachen - Beiträge zum 1. Arbeitskreis in Berlin (12.-14.9.2016)

(C) 2018 innsbruck university press, ISBN 978-3-903187-11-5, DOI 10.15203/3187-11-5 
legungen, die sich nicht bedeutend unterscheiden, unter der Berücksichtigung der unterrichtlichen Bedingungen zusammenzuführen, in denen der Witz mündlich als Redegattung sowie schriftlich als Textsorte vorkommt. Des Weiteren sind zwei definitorische Auffassungen von Bedeutung: Der Witz im engeren Sinne als анекдоm stellt eine kurze, pointierte Textsorte/Redegattung dar. Der Witz im weiteren Sinne umfasst alle kurzen Texte, die ein komisches bzw. humoristisches Moment enthalten. Ausgehend von diesen Definitionen kann eine Untersuchung von Witzen im Unterrichtskontext strukturiert erfolgen.

\section{Forschungsüberblick}

Die wissenschaftliche Auseinandersetzung mit dem Witz blickt auf eine lange Geschichte zurück. Die Perspektiven, aus denen der Witz untersucht wurde, decken dabei ein besonders breites Spektrum ab: Es existieren zahlreiche philosophisch-ästhetische, psychologische, anthropologisch-soziologische, linguistische, literatur- und kulturwissenschaftliche Untersuchungen (vgl. Best 1989, Freud 1905, Lipps 1898, Marfurt 1977, Plessner 1941, Wellek 1949). Diese Studien verlaufen meist transdisziplinär, was der Komplexität des Witzes als Untersuchungsgegenstand geschuldet ist. Dabei spielt insb. die Theorie des Komischen bzw. des Humors eine bedeutende Rolle.

Der Witz in Zusammenhang mit dem Komischen wird in mehreren kulturwissenschaftlich orientierten Studien unter diversen Gesichtspunkten behandelt: Dabei stehen die interkulturelle Wirksamkeit und Repräsentanz des Witzes (vgl. u. a. Dimova 2002), die Beziehung zwischen Witz und Wirklichkeit als Form der ästhetischen Weltaneignung (vgl. u. a. Jakobi \& Waldschmidt 2015) oder kulturspezifische Aspekte von Humor im Rahmen der interkulturellen Kommunikation (vgl. Stojić \& Žagar-Šoštarić 2015) im Fokus.

Die literaturgeschichtliche Forschung im deutschsprachigen Raum zeigt bereits im 18. Jahrhundert besonderes Interesse am Witz (vgl. u. a. Winkler \& Goulding 2005). Die Untersuchung des Witzes seitens der Literaturwissenschaft, in der der Witz aus dem Komischen hergeleitet wird, zeigt sich in diversen Versuchen, die wesentliche Komponente des Witzes, die Pointe, zu definieren. So betont der

Anka Bergmann, Olga Caspers \& Wolfgang Stadler (Hg.)

Didaktik der slawischen Sprachen - Beiträge zum 1. Arbeitskreis in Berlin (12.-14.9.2016)

(C) 2018 innsbruck university press, ISBN 978-3-903187-11-5, DOI 10.15203/3187-11-5 
deutsche Literaturwissenschaftler Preisendanz (1970: 18) bereits am Anfang der 1970er Jahre die Pointe als das Unterscheidungsmerkmal, das den Witz von „vielerlei Sorten komischer oder spaßhafter Histörchen und Äußerungen“ abgrenzt.

Es wurden bislang überschaubare Versuche unternommen, den Witz im Rahmen der Sprachendidaktik zu beschreiben. Der Schwerpunkt liegt dabei auf dem erstsprachlichen Unterricht. So geht Ulrich (1980) auf die vielfältigen Möglichkeiten einer didaktischen Behandlung des Witzes im Deutschunterricht ein. Auf der Basis der Theorie des Witzes geht der Wissenschaftler auf die Sprech- und Schreibimpulse, Strukturen des Witzes sowie die Witzrezeption und -produktion in der Muttersprache ein.

Die Fremdsprachendidaktik als eine Integrationswissenschaft, die Theorie und Lehre in einem verkörpert (vgl. Lembens \& Peschek 2009), ,greift sowohl inhaltlich als auch forschungsmethodisch auf Bezugswissenschaften zurück" (Caspari 2016: 12), um gelenkten Spracherwerb zu erforschen und zu optimieren. Die Bezugsdisziplinen der Fachdidaktik des Russischen, die Linguistik, die Literaturwissenschaft, die Kulturwissenschaften sowie die Erziehungswissenschaft und die Unterrichtsforschung liefern viele ertragreiche Ergebnisse, die ich als Anknüpfungspunkte für die fachdidaktische Erforschung des Witzes betrachte. Fachwissenschaftliche Forschung bildet also Basis und Ausgangspunkt der fachdidaktischen Forschung, kann diese aber nicht ersetzen.

Ueda (2013) veröffentlicht seine Überlegungen über die Textsorte „Witz“ im Deutschunterricht in Japan, dabei stehen linguistische Ansätze zum Philosophieren mit Kindern im Fokus. Diese philosophisch ausgerichtete Monographie, die auf den Erfahrungen des Autors basiert, liefert inspirierende Impulse für die weitere Erforschung des Themas „Witz im Fremdsprachenunterricht“.

Die Erforschung des Humors im Kontext des Fremdsprachenlernens erfolgt in der Gegenwart vorwiegend aus linguistischer Perspektive. In den letzten 30 Jahren ist eine Reihe von linguistischen Werken zum Thema Humor erschienen, die den Witz fokussiert beschreiben und analysieren. Raskin (1985) revolutioniert den Zugang zum Witz aus linguistischer Perspektive mit seiner Semantic-Script Theory of Humor, die sich mit dem Witz, ,the simplest and least complicated type of humorous text" (Attardo 2008: 108), auseinandersetzt. Die allgemeine Theorie des verbalen Humors von Attardo \& Raskin (1991) legt den Grundstein für

Anka Bergmann, Olga Caspers \& Wolfgang Stadler (Hg.)

Didaktik der slawischen Sprachen - Beiträge zum 1. Arbeitskreis in Berlin (12.-14.9.2016)

(C) 2018 innsbruck university press, ISBN 978-3-903187-11-5, DOI 10.15203/3187-11-5 
eine breitere sprachwissenschaftliche Erforschung des Humors, bei der der Witz im Fokus bleibt. Eine Reihe von Untersuchungen befasst sich mit Fragestellungen der Pragmatik und zeigt Mechanismen des Erkennens, Verstehens und der Produktion des Humors in einer Fremdsprache auf (Kagan 2016, Kusnetsova 2015, Shardakova 2010, 2013, 2016).

Der Untersuchungsbereich „Humor im Klassenzimmer bzw. im Fremdsprachenunterricht" genießt große Aufmerksamkeit in der gegenwärtigen Forschung. Der Sammelband Humor im Fremdsprachenunterricht (Löschmann 2015) durchleuchtet „linguistische, psychologische, interkulturelle und translationswissenschaftliche Aspekte des Humors [...], um effiziente Einsatzmöglichkeiten im Fremdsprachenunterricht aufzuzeigen" (ebd.: Cover). Der Band ist in der Reihe „Deutsch als Fremdsprache in der Diskussion“ veröffentlicht. Die Beiträge in diesem Band stammen aus fünf Ländern - aus Deutschland, Kanada, Kroatien, Russland und den USA, was auf die Bedeutung dieser Forschungsrichtung weltweit hinweist. Nicht ein Beitrag jedoch widmet sich explizit der Textsorte „Witz“ im Fremdsprachenunterricht, obwohl die meisten der Aufsätze gewisse Hinweise bzw. sogar ausführliche Überlegungen zur Analyse, Klassifizierung und zu Formen des Witzes enthalten (vgl. Cui 2015, Erofeev 2015, Kusnetsova 2015). Die Monographie Humor in the Classroom (Bell \& Pomerantz 2016) bietet praktische, forschungsbasierte Antworten auf die Fragen der Unterrichtsforschung und der Fremdsprachendidaktik in Bezug auf die kognitiven Vorteile, die der Humor im L2-Klassenzimmer-Diskurs erlaubt und fördert.

Diese zwei Publikationen zeigen, dass die fachdidaktische Witzforschung eine mehr oder weniger fokussierte Konkretisierung im Rahmen des Forschungsfeldes „Humor im Fremdsprachenunterricht“ erfährt. Das breite Spektrum dieses Bereichs veranschaulicht das Zitat aus dem Vorwort des Herausgebers: „Indem wir Humor als Oberbegriff für alle Arten von Texten, Ereignissen, Handlungen, auch nonverbalen Erscheinungsformen wählen, die zum Lachen, zu Spaß und Erheiterung im Unterricht führen können, ziehen wir den Kreis um den Humor so groß, dass es in der Tat schwer fällt, ihn per definitionem zu fassen“ (Löschmann 2015: 15). In dieser Breite des Forschungsfeldes Humor wird die Witzforschung trotz ihrer Präsenz insofern vernachlässigt, als auf sie nicht präzise eingegangen wird.

Anka Bergmann, Olga Caspers \& Wolfgang Stadler (Hg.)

Didaktik der slawischen Sprachen - Beiträge zum 1. Arbeitskreis in Berlin (12.-14.9.2016)

(C) 2018 innsbruck university press, ISBN 978-3-903187-11-5, DOI 10.15203/3187-11-5 
Im Rahmen des Forschungsfeldes „Lachen im Unterricht“ werden Witze aus der Perspektive der Unterrichtsforschung analysiert. Die positive Atmosphäre, die dank einer lustigen Kommunikation zwischen den UnterrichtsteilnehmerInnen geschaffen wird, trägt zur Lernmotivation bei und wirkt sich positiv auf die Lernergebnisse aus (vgl. Junko \& Ohta 2008). Dabei visieren die Forscherinnen Witze nicht als Textsorte oder Redegattung, sondern fokussieren auf spontane Aussagen und Situationen, die im Unterricht stattfinden.

Der oben angeführte Forschungsüberblick weist deutliche Desiderate in Bezug auf die fachdidaktisch ausgerichtete Witzforschung im Fremdsprachenunterricht auf. Eine systematische Studie über Witze im Fremdsprachenunterricht, konkret in Bezug auf das Russische, fehlt. Eine umfassende Erforschung des Potenzials von Witzen im Unterricht sollte sowohl theoretische als auch praktische Ziele verfolgen. Die theoretischen Ziele fremdsprachendidaktischer Forschung umfassen die Auseinandersetzung mit einzelnen Faktoren des fremdsprachlichen Lernens und Lehrens, die praktischen Ziele streben eine Verbesserung der Qualität des Fremdsprachenunterrichts an (Caspari 2016: 12).

\section{Perspektiven der Witzforschung zu subjektiven Haltungen der UnterrichtsteilnehmerInnen}

Fremdsprachendidaktische Forschung unterliegt im 21. Jahrhundert einer thematischen Schwerpunktwandlung: Der thematische Fokus verschiebt sich seit dem Beginn der 2000er Jahre von der Inhaltsperspektive auf die Lernerperspektive (vgl. Klippel 2016). Der Witz erlaubt aber, die beiden Perspektiven zu vereinen, denn er gehört sowohl zum Inhalt des Unterrichts als auch zum Bereich des unterrichtlichen Handelns, in dem funktionale Sprachfertigkeiten der Lernenden sowie die subjektive Haltung der Lehrpersonen zu Witzen im Fokus stehen.

In einem an der Universität Salzburg laufenden Projekt wird der Einsatz von Witzen im Russischunterricht unter zwei Gesichtspunkten erforscht. Zum einen werden gegenwärtige Lehrwerke für Russisch hinsichtlich der Präsenz von Witzen analysiert, zum anderen wird die subjektive Haltung von Lehrenden zu Witzen im Unterricht erkundet. Diese zwei Ausrichtungen der Witzforschung

Anka Bergmann, Olga Caspers \& Wolfgang Stadler (Hg.)

Didaktik der slawischen Sprachen - Beiträge zum 1. Arbeitskreis in Berlin (12.-14.9.2016)

(C) 2018 innsbruck university press, ISBN 978-3-903187-11-5, DOI 10.15203/3187-11-5 
erlauben die den Unterricht beeinflussenden Faktoren wie Lehrwerke und Lehrpersonen in die Erforschung des Witzes miteinzubeziehen.

Wie bereits in der Einleitung angedeutet, können Lehrwerke eine Auskunft über den Einsatz des Witzes im Russischunterricht geben. Die zentrale Stellung des Lehrwerks im Fremdsprachenunterricht (vgl. Fäcke 2017: 35) begründet die Analyse der Inhalte von Lehrwerken hinsichtlich verschiedener Arten von Witzen. Dabei können die Lehrwerke sowohl unter qualitativen als auch quantitativen Gesichtspunkten analysiert werden. Folgende Fragen bilden die Basis der Analyse: Kommen Witze im Lehrwerk vor und wenn ja, wie oft? Welche Funktionen erfüllen die Witze? Gibt es einen Zusammenhang zwischen der Witzfrequenz und dem Sprachniveau bzw. der Progression des Lehrwerks? Welche Aufgaben sind in Bezug auf Witze zu finden? Erlauben Witze Rückschlüsse auf gesellschaftliche und kulturelle Normen und Werte?

Das Wissen über Kognitionen der Selbst- und Weltsicht respektive des Einsatzes von Witzen kann zu einem besseren Verständnis unterrichtlicher Prozesse führen. Die Erforschung subjektiver Theorien von Individuen bezieht sich auf Konstrukte, die aus „Wissen, Wertungen, erinnerten Erfahrungen, Schlussfolgerungen und Absichten bestehen" (Caspari 2003: 70). Dieser Forschungsansatz zu subjektiven Theorien, der das „am weitesten ausgearbeitete Konzept“ (ebd.) bietet, geht von einem epistemologischen Subjektmodell aus, wonach das Individuum seine internen Prozesse, Sinn- und Bedeutungsstrukturen sowie Handlungen reflektieren kann. Der Mensch als Erkenntnisobjekt ist in der Lage „sich von seiner Umwelt zu distanzieren und unabhängig zu machen, indem es sie mithilfe selbst konstruierter Kategorien beschreibt, erklärt und mit Bedeutung versieht" (Groeben et al. 1988: 16). Darüber hinaus wird das Individuum in „Parallelität zum Selbstbild des Wissenschaftlers" (ebd.) gesehen. Analog zu wissenschaftlichen Theorien dienen subjektive Theorien „der Erklärung, Prognose und Technologie“ in Bezug auf einen bestimmten Gegenstand und können folgende Elemente umfassen: subjektive Konstrukte, subjektive Daten, subjektive Wenn-Dann-Hypothesen, sowie affektive Aspekte und Einstellungen (vgl. Caspari 2003: 78).

Das Ziel ist es, subjektive Theorien der Lehrenden zum Einsatz von Witzen im Unterricht zu erkunden. Dabei soll geklärt werden, wie der Witz seitens der Lehrenden im Unterricht wahrgenommen wird, welche Erwartungen bzw. Er-

Anka Bergmann, Olga Caspers \& Wolfgang Stadler (Hg.)

Didaktik der slawischen Sprachen - Beiträge zum 1. Arbeitskreis in Berlin (12.-14.9.2016)

(C) 2018 innsbruck university press, ISBN 978-3-903187-11-5, DOI 10.15203/3187-11-5 
fahrungen Lehrende im Zusammenhang mit dem Witz haben. Kennen die Lehrpersonen die Vorstellungen der SchülerInnen zum Thema Witz und wenn sie den Witz in ihre Unterrichtsplanungen einbinden, in welcher Weise geschieht dies?

Das Zusammenschließen dieser zwei Forschungsteilbereiche sollte neue Einblicke in die Gestaltung des Fremdsprachenunterrichts sowie in die Kognitionsprozesse der UnterrichtsteilnehmerInnen geben. Im Folgenden wird eine Pilotstudie vorgestellt, die das Potenzial des Witzes im Russischunterricht verdeutlicht.

\section{Pilotierung der Fragebogenstudie zu den Einstellungen von Lernenden zum Witz im Unterricht}

Die Pilotstudie wurde mit dem Ziel konzipiert, die subjektive Haltung der Lernenden in Bezug auf den Witz im Unterricht zu eruieren. Die Umfrage war an die Studierenden aller Russischkurse, die im Wintersemester 2016/17 an der Universität Salzburg abgehalten wurden, gerichtet. Es wurden insgesamt 45 Fragebögen (11 Männer, 28 Frauen und 6 Personen ohne Angabe) erfasst. Eine genauere Beschreibung der ProbandInnen-Gruppe soll hier nicht vorgenommen werden, da es sich um eine Pilotstudie handelt. Die Pilotstudie enthält 25 Aussagen und Fragen, die in drei Kategorien organisiert sind. Die erste Kategorie enthält zehn Aussagen und eine Frage zum Einsatz des Witzes im Russischunterricht, die zweite Kategorie der Aussagen geht auf die Selbsteinschätzung bezüglich der Kompetenz, Witze zu verstehen, ein, und die dritte Kategorie umfasst offene Fragen zur Lernbiographie jedes/jeder Befragten.

\subsection{Aufbau des Fragebogens}

5.1.1 Kategorie 1: Items zum Einsatz von Witzen im Russischunterricht

Den Studierenden wurden in dieser ersten Kategorie zehn von der Forscherin formulierte Aussagen vorgelegt, für die eine Likert-Skala verwendet wurde. Die Aussagen sollen die Einstellungen der Lernenden zu drei Fertigkeiten (Sprechen bzw. Erzählen, Lesen und Hören) in Bezug auf den Witz in ihrer Muttersprache und 
in der Zielsprache Russisch festhalten. Sie sind handlungsorientiert formuliert, z. B. „Ich erzähle gern Witze in meiner Muttersprache“. Die Verbindung zwischen dem Interesse am Gegenstand bzw. der Lernmotivation und dem Lernerfolg steht im Fokus mehrerer pädagogisch-psychologischer Studien (vgl. u. a. Hoffmann 2014) und ist für die Planung und „optimale Gestaltung des Unterrichts [...] ein wichtiger Aspekt" (Krapp 1996: 105). Die Aussagen dieser Kategorie des Fragebogens sollen die subjektive Wahrnehmung des Witzes hinsichtlich seines Einflusses auf die Lernatmosphäre veranschaulichen. Zuletzt wurde die subjektive Häufigkeit des Witzeinsatzes abgefragt. Die folgenden Hypothesen wurden überprüft:

1. Die Haltung zum Witz in Bezug auf die drei Fertigkeiten (Sprechen, Lesen, Hören) in der Muttersprache spiegelt sich in der Haltung zum Witz in der Zielsprache Russisch wider.

2. Die subjektive Meinung der Lernenden ist, dass Witze in der Zielsprache die Lernatmosphäre positiv beeinflussen.

3. Der Witz kommt im Russischunterricht selten vor.

\subsubsection{Kategorie 2: Aussagen zum Verständnis von Witzen}

Die Aussagen der zweiten Kategorie gehen auf den Begriff des Witzes bzw. das Verstehen dieses Begriffs sowie auf unterschiedliche Arten von Witzen ein. Die Formulierung der Aussagen dieser Kategorie geht von einer weiten Definition des Witzes aus, die nicht nur pointierte Texte, sondern alle Texte, die in Verbindung mit Lachen und Humor stehen, umfasst. Außerdem überprüfen die Aussagen die subjektive Verständlichkeit von Witzen in der russischen Sprache in Abhängigkeit von verschiedenen Arten von Humor, dem verbalen und dem referenziellen (vgl. Attardo 1994). Referenzielle Witze, die als leichter übersetzbar gelten (ebd.), wurden zusätzlich - unterteilt in zwei Kategorien - angeboten: Witze, die sich auf das Allgemeinwissen gegenüber Witzen, die sich auf kulturelle Besonderheiten Russlands beziehen (universal vs. culture-based). Dazu wurden den ProbandInnen die entsprechend formulierten Aussagesätze vorgelegt: „Ich verstehe russischsprachige Witze, die sich auf das Allgemeinwissen beziehen“, „Ich verstehe

Anka Bergmann, Olga Caspers \& Wolfgang Stadler (Hg.)

Didaktik der slawischen Sprachen - Beiträge zum 1. Arbeitskreis in Berlin (12.-14.9.2016)

(C) 2018 innsbruck university press, ISBN 978-3-903187-11-5, DOI 10.15203/3187-11-5 
russischsprachige Witze, die sich auf kulturelle Besonderheiten Russlands beziehen“, „Ich verstehe russischsprachige Witze, die sich auf sprachliche Besonderheiten des Russischen beziehen “. Folgende Hypothesen werden dabei überprüft:

1. Unter dem Begriff „Witz“ werden kurze Texte mit einer Pointe verstanden.

2. Russischsprachige Witze, die sich auf das Allgemeinwissen beziehen, werden besser verstanden.

3. Russischsprachige Witze, die sich auf kulturelle Besonderheiten Russlands beziehen, werden im Vergleich zur ersten Kategorie (Allgemeinwissen) schwerer verstanden.

4. Russischsprachige Witze, die sich auf sprachliche Besonderheiten des Russischen beziehen, werden am schwersten verstanden.

5. Das Verstehen des russischsprachigen Witzes stellt für Lernende eine subjektiv empfundene Herausforderung dar.

\subsubsection{Kategorie 3: Fragen zu Lernerbiographien}

Die dritte Kategorie umfasst Fragen zu Lern- und Sprachbiographien der befragten Studierenden. Nach der L1 und der Anzahl der in der Schule und an der Universität gelernten bzw. zu lernenden Sprachen wird auch die Anzahl der Semester, in denen Russisch gelernt wurde, abgefragt. Das Ziel ist es, einen $\mathrm{Zu}-$ sammenhang zwischen der Mehrsprachigkeit der Studierenden sowie der Dauer der Auseinandersetzung mit dem Russischen und dem subjektiv empfundenen Verstehen russischsprachiger Witze zu überprüfen.

\subsection{Ergebnisse}

\subsubsection{Einsatz des Witzes im Russischunterricht}

Die Überprüfung der Hypothesen dieser Kategorie bringt folgende Erkenntnisse ans Licht. Die erste Hypothese hat sich dabei nicht gänzlich bestätigt. 26,7\% stimmen der Aussage „Ich erzähle gern Witze in meiner Muttersprache“ zu, je 28,9\% der Befragten wählen „trifft eher nicht zu“ und „trifft eher zu“. Demgegenüber 
steht eine eindeutig ablehnende Haltung zum Erzählen russischsprachiger Witze: 48,9\% wählen „trifft nicht zu“ und 44,4\% „trifft eher nicht zu“. Eine Gegenüberstellung der Fertigkeiten, Witze in der L1 und in der russischen Sprache zu hören, die aus den Aussagen „Ich höre gern Witze in meiner Muttersprache/Ich höre gern russischsprachige Witze im Unterricht" hervorgeht, bringt aber überraschende Ergebnisse zutage: Der Prozentanteil in der Kategorie „trifft eher zu“ ist in beiden Sprachen gleich und beträgt 42,2\%, wobei auch die Kategorie „trifft zu“ von 46,7\% für die L1 und 40,0\% für Russisch gewählt wird. Für die Fertigkeit Lesen sind die höchsten Prozentzahlen wie folgt verteilt: 35,6\% wählen für Witze in ihrer L1 die Kategorie „trifft eher zu“ und 42,2\% wählen dieselbe Kategorie für russischsprachige Witze. Die rezeptiven Fertigkeiten Hören und Lesen erfreuen sich somit im Vergleich zum Erzählen größerer Beliebtheit in Bezug auf den Witz im Unterricht. Das kann aber auch damit zusammenhängen, dass die Befragten aus unterschiedlichen Gründen nicht in der Lage oder Stimmung sind, selbst Witze zu erzählen. Die subjektive Haltung zum Witz in Bezug auf diese zwei Bereiche in der L1 spiegelt sich in der Haltung zum russischen Witz wider.

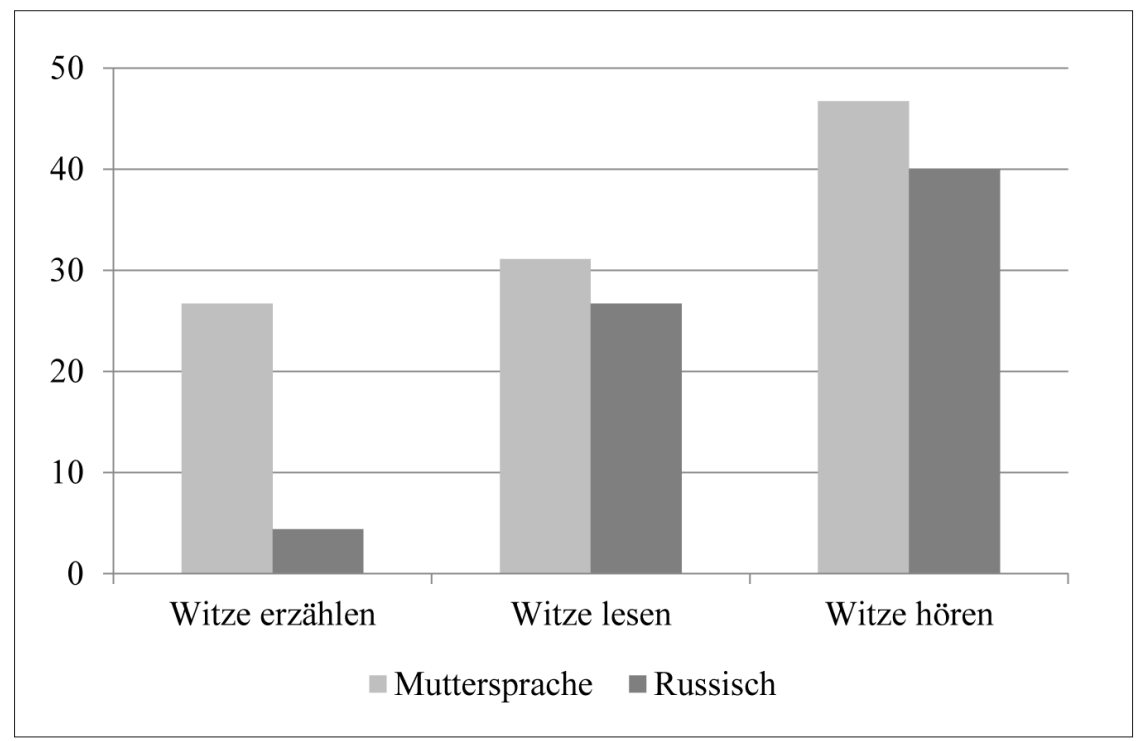

Abbildung 2: Haltung zum Witz in der Muttersprache und im Russischen Häufigkeiten in \% 
Die zweite Hypothese, dass der russischsprachige Witz in der subjektiven Wahrnehmung der Lernenden die Lernatmosphäre positiv beeinflusst, wurde bestätigt. Für die Aussage „Ich bin der Meinung, dass russischsprachige Witze die Lernatmosphäre positiv beeinflussen“ wurden ausschließlich positive Kategorien gewählt: 33,3\% für „trifft eher zu“ und 57,8\% für „trifft zu“. Die übrigen 10\% haben keine Angaben zu dieser Frage gemacht.

Die dritte Hypothese der ersten Kategorie, dass der Witz im Unterricht selten eingesetzt wird, wurde durch die Frage „Wie häufig wurde Ihrer Schätzung nach der russische Witz in Ihrer Lernvergangenheit im Russischunterricht eingesetzt?“ überprüft. 71,1\% der Befragten beantworten die Frage mit der Auswahl „eher selten“. Daraus kann geschlussfolgert werden, dass der Witz im Russischunterricht in der Tat selten vorkommt, was wiederum die Hypothese bestätigt.

\subsubsection{Verständnis von Witzen}

Die erste Aussage dieser Kategorie hält die subjektive Auffassung des Begriffs „Witz“ fest. Den Studierenden wurden vier Aussagen zur Definition des Witzes sowie eine Möglichkeit, eine eigene Definition zu geben, angeboten (s. Tab. 1). Mehrfachantworten waren erlaubt. Dabei formen sich zwei Kategorien als charakteristische Merkmale des Witzes in der subjektiven Auffassung dieses Begriffs seitens der Befragten: 53,3\% verbinden den Witz mit Humor und 60,0\% halten eine Pointe für notwendig. Die Kategorien „Alles, was mich zum Lachen bringt“ und „ein kurzer lustiger Text“ werden von je 37,8\% als wesentlich für die Definition von Witzen wahrgenommen. Somit gilt die erste Hypothese als bestätigt.

Die Überprüfung der Hypothesen 2 bis 4 zeigt, dass der Schwierigkeitsgrad des subjektiven Verstehens russischsprachiger Witze wie folgt abnimmt: Witze, die sich auf das Allgemeinwissen beziehen, werden von 55,6\% „eher häufig“ verstanden, Witze, die sich auf kulturelle Besonderheiten Russlands beziehen, werden von $40,0 \%$ der Befragten „eher selten“ und von $31,1 \%$ „eher häufig“ verstanden, und Witze, die sich auf sprachliche Besonderheiten des Russischen beziehen, werden von $40,0 \%$,eher selten“ und von $26,7 \%$ „nie“verstanden. So-

Anka Bergmann, Olga Caspers \& Wolfgang Stadler (Hg.)

Didaktik der slawischen Sprachen - Beiträge zum 1. Arbeitskreis in Berlin (12.-14.9.2016)

(C) 2018 innsbruck university press, ISBN 978-3-903187-11-5, DOI 10.15203/3187-11-5 
mit bestätigen die Ergebnisse die in den Hypothesen angenommene Taxonomie der Schwierigkeit des Verständnisses.

Tabelle 1: Angabe wesentlicher Parameter bei der Definition von Witzen

\begin{tabular}{|l|c|c|c|}
\hline \multicolumn{3}{|c|}{ Definition von Witz } \\
\hline & $N$ & $\%$ & $\%$ der Fälle \\
\cline { 2 - 4 } & 17 & 19,1 & 37,8 \\
\hline $\begin{array}{l}\text { Witz ist alles, was mich zum } \\
\text { Lachen bringt }\end{array}$ & 24 & 27,0 & 53,3 \\
\hline $\begin{array}{l}\text { Witz ist alles, was mit Humor } \\
\text { in Verbindung steht }\end{array}$ & 17 & 19,1 & 37,8 \\
\hline Witz ist ein kurzer lustiger Text & 27 & 30,3 & 60,0 \\
\hline Witz enthält eine Pointe & 4 & 4,5 & 8,9 \\
\hline Eigene Definition des Witzes & 89 & 100,0 & 197,8 \\
\hline Gesamt & & & \\
\hline
\end{tabular}

Die letzte Hypothese für diese Kategorie zum subjektiven Verstehen des russischsprachigen Witzes wird durch die Aussage „Ich verstehe russischsprachige Witze“ überprüft (Tab. 2).

Tabelle 2: Subjektive Einschätzung der Herausforderung, Witze im Russischen zu verstehen

\begin{tabular}{|l|c|c|}
\hline \multicolumn{3}{|c|}{ Ich verstehe russischsprachige Witze ... } \\
\hline & $N$ & $\%$ \\
\hline nie & 5 & 11,1 \\
\hline eher selten & 18 & 40,0 \\
\hline eher häufig & 14 & 31,1 \\
\hline sehr häufig & 8 & 17,8 \\
\hline Gesamt & 45 & 100,0 \\
\hline
\end{tabular}


40,0\% der Befragten geben an, Witze eher selten zu verstehen, 31,1\% wählen „eher häufig“. Daraus kann man schließen, dass der Witz zwar eine Herausforderung darstellt, diese aus der Sicht der Lernenden aber überwindbar ist. Bei einer Gegenüberstellung der zusammengesetzten positiven („eher häufig“ und „sehr häufig“) und negativen („nie“ und „eher selten“) Kategorien, 48,9\% gegenüber $51,1 \%$, ist das Verhältnis aber relativ ausgeglichen, was eine eindeutige Interpretation ausschließt.

\subsubsection{Lernerbiographien}

Die Auswertung des Zusammenhangs zwischen dem subjektiven Verstehen von Witzen und der Anzahl der Semester, in denen Russisch gelernt wurde, zeigt interessante Verbindungen. Dennoch werden diese Ergebnisse als nicht repräsentativ gedeutet, da die Fragebögen vorwiegend von Russisch-AnfängerInnen ausgefüllt wurden. Für einen Reliabilitätsanspruch dieses Teils bedarf es einer größeren Studie, die sämtliche Studierendengruppen umfassend abdeckt, was im Rahmen dieser Pilotstudie nicht geleistet werden konnte.

\section{Zusammenfassung und Ausblick}

Die Ergebnisse der Pilotstudie sind für die Witzforschung insofern von Bedeutung, als sie die Haltung der Lernenden zum Witz als auch ihr Witzverständnis beleuchten. So sind sich die Lernenden des Russischen der Herausforderungen des Witzes im Unterricht bewusst, dennoch stehen sie dem Witz positiv gegenüber. Es ist anzumerken, dass die Fragebogenstudie die allgemein geltenden Prinzipien des Fremdsprachenunterrichts bestätigt. Die dem Grad der Handlungsorientiertung angemessene Progression des Einsatzes des Witzes im Unterricht sollte dem Grad der Willigkeit der Auseinandersetzung mit dem Witz folgen, sodass zunächst die rezeptiven Fertigkeiten aufgebaut werden und eventuell ein leichterer Übergang zur produktiven Fertigkeit des Sprechens entstehen kann. Genauso verhält es sich mit den verschiedenen Arten des Witzes: Die am leichtesten zu verstehenden Arten sollen vor den schwierigeren im Unterricht eingesetzt werden.

Anka Bergmann, Olga Caspers \& Wolfgang Stadler (Hg.)

Didaktik der slawischen Sprachen - Beiträge zum 1. Arbeitskreis in Berlin (12.-14.9.2016)

(C) 2018 innsbruck university press, ISBN 978-3-903187-11-5, DOI 10.15203/3187-11-5 
Die Pilotstudie hat auch gezeigt, dass manche Items des Fragebogens wegen der sich überschneidenden Formulierung (vgl. Kategorie 1, Items 9 und 10) nur z. T. ausgewertet werden konnten. So wurde Item 10 aus der Auswertung ausgeschlossen. Außerdem sind bei der Auswertung der Items Einschränkungen in der Interpretation der Ergebnisse deutlich geworden, wie bspw. bei der subjektiven Einschätzung der Herausforderung, Witze im Russischen zu verstehen. Der Einsatz von anderen Instrumenten zur Erfassung subjektiver Theorien, wie bspw. eine Interviewstudie könnte hier mehr Klarheit mit sich bringen.

Eine systematische fachdidaktische Auseinandersetzung mit dem Witz kann dazu beitragen, dass das Potenzial des Witzes für den Russischunterricht ausgelotet wird. Die Erforschung kognitiver Prozesse der UnterrichtsteilnehmerInnen, ihrer subjektiven Menschenbildannahmen und Kognitionen der Selbst- und Weltsicht in Bezug auf Witze als Textsorte und Redegattung im Unterricht sowie der Präsenz und Vielfalt von Witzen in Lehrwerken soll neue Facetten des Russischunterrichts ans Licht bringen.

Ein weiterer Forschungsbereich ergibt sich aus der Frage, inwiefern sich Witze im Fremdsprachenunterricht für kulturelles Lernen eignen. Die interkulturelle Dimension von Witzen hebt die Bedeutung dieser Textsorte für den fremdsprachlichen Unterricht hervor. Witze als Bestandteil mündlicher und schriftlicher Kommunikation im Unterricht sind nicht nur Indikatoren sprachlicher Kompetenz, sondern Träger interkultureller Inhalte. Witze enthalten Aussagen über gesellschaftliche Stereotype, gesellschaftliche Realität und eine ganze Reihe gesellschaftlicher Abhandlungen und Abläufe.

\section{Literaturverzeichnis}

Attardo, S. (2008). A Primer for the Linguistics of Humor. In V. Raskin (Hrsg.), The Primer of Humor Research (101-156). Berlin: de Gruyter.

Attardo, S. \& Raskin, V. (1991). Script Theory Revis(it)ed: Joke Similarity and Joke Representation Model. HUMOR: International Journal of Humor Research, 4 (3/4), 293-347.

Attardo, S. (1994). Linguistic Theories of Humor. Berlin: de Gruyter.

Anka Bergmann, Olga Caspers \& Wolfgang Stadler (Hg.)

Didaktik der slawischen Sprachen - Beiträge zum 1. Arbeitskreis in Berlin (12.-14.9.2016)

(C) 2018 innsbruck university press, ISBN 978-3-903187-11-5, DOI 10.15203/3187-11-5 
Bell, N. D. \& Pomerantz, A. (2016). Humor in the Classroom. A Guide for Language Teachers and Educational Researches. New York, London: Routledge.

Best, O. F. (1989). Der Witz als Erkenntnis Kraft und Formprinzip. Darmstadt: Wissenschaftliche Buchgesellschaft.

Caspari, D. (2003). Fremdsprachenlehreinnen und Fremdsprachenlehrer. Studien zu ihrem beruflichen Selbstverständnis. Tübingen: Narr.

Caspari, D. (2016). Grundfragen fremdsprachendidaktischer Forschung. In D. Caspari et al. (Hrsg.), Forschungsmethoden in der Fremdsprachendidaktik. Ein Handbuch (7-22). Tübingen: Narr.

Chimik, V. (2002). Anekdot kak unikal'noe javlenie russkoj rečevoj kul'tury. In M. Kagan \& E. Sokolov (Hrsg.), Anekdot kak fenomen kul'tury (17-31). Sankt-Peterburg: Sankt-Peterburgskoe filosofskoe obščestvo.

Cui, P. (2015). Deutscher und chinesischer Humor - ein Vergleich. In M. Löschmann (Hrsg.), Humor im Fremdsprachenunterricht (59-73). Frankfurt a. M.: Peter Lang.

Dement'ev, V. (2010). Teorija rečevych žanrov. Moskau: Znak.

Dimova, A. (2002). Aphorismus und Witz interkulturell. In M. Auer, U. Mueller (Hrsg.), Kanon und Text in interkulturellen Perspektiven: andere Texte anders lesen (199-213). Stuttgart: Verlag Hans-Dieter Heinz Akademischer Verlag.

Efremova, T. (2006). Sovremennyj tolkovyj slovar' russkogo jazyka v trech tomach. Moskau: Astrel' AST.

Erofeev, J. (2015). Verbaler Chauvinismus in deutschsprachigen Frauenwitzen im Internet. In M. Löschmann (Hrsg.), Humor im Fremdsprachenunterricht (133-144). Frankfurt a. M.: Peter Lang.

Fäcke, Ch. (2016). Lehrwerkforschung - Lehrwerkgestaltung - Lehrwerkrezeption. Überlegungen zur Relevanz von Lehrwerken für den Fremdsprachenunterricht. In. M. Rückl (Hrsg.), Sprachen und Kulturen vermitteln und vernetzen (34-49). Münster, New York: Waxsmann.

Freud, S. (1999). Der Witz und seine Beziehung zum Unbewussten. In S. Freud, Gesammelte Werke. (Band 6). London, Frankfurt a. M.: S. Fischer Verlag.

GeR=Trim, J., North, B. \& Coste, D. (Hrsg.) (2001). Gemeinsamer europäischer Referenzrahmen für Sprachen: Lernen, lehren, beurteilen. Berlin: Langenscheidt.

Groeben, N. et al. (1988). Forschungsprogramm subjektive Theorien. Eine Einführung in die Psychologie des reflexiven Subjekts. Tübingen: Francke Verlag.

Anka Bergmann, Olga Caspers \& Wolfgang Stadler (Hg.)

Didaktik der slawischen Sprachen - Beiträge zum 1. Arbeitskreis in Berlin (12.-14.9.2016)

(C) 2018 innsbruck university press, ISBN 978-3-903187-11-5, DOI 10.15203/3187-11-5 
Hoffmann, S. (2014). Mündliche Kompetenz und Bewusstsein beim unterrichtlichen Fremdsprachenlernen. Tübingen: Narr.

Jakobi, C. \& Waldschmidt, Ch. (2015). Witz und Wirklichkeit. Komik als Form ästhetischer Weltaneignung. Bielefeld: Transcript.

Junko, M. \& Ohta, A. S. (Hrsg.) (2008). Japanese Applied Linguistics: Discourse and Social Perspectives. New York: Continuum.

Kagan, O. (2016). Structural Ambiguity in Russian Humor Creation. Russian Linguist, 40, 1-10.

Kotthoff, H. (2011). Besondere Formen des Erzählens in Interaktionen: Vom Klatsch über den Bericht bis zum Witz und spaßigen Fantasien. In: S. Habscheid (Hrsg.), Textsorten, Handlungsmuster, Oberflächen. Linguistische Typologie in der Kommunikation (389-413). Berlin: de Gruyter.

Klippel, F. (2016). Forschungstraditionen der Fremdsprachendidaktik. In D. Caspari et al. (Hrsg.), Forschungsmethoden der Fremdsprachendidaktik. Ein Handbuch (23-31). Tübingen: Narr.

Krapp, A. (1996). Die Bedeutung von Interesse und intrinsischer Motivation für den Erfolg und die Steuerung des schulischen Lernens. In G. Schnaitmann (Hrsg.), Theorie und Praxis der Unterrichtsforschung. Methodologische und praktische Ansätze zur Erforschung von Lernprozessen (87-110). Donauwörth: Auer Verlag.

Kurganov, E. (2015). Anekdot kak žanr russkoj slovesnosti. Moskau: Arsis Books.

Kusnetsova, N. (2015). Linguistische Aspekte beim Einsatz von Komik und Humor im Fremdsprachenunterricht. In M. Löschmann (Hrsg.), Humor im Fremdsprachenunterricht (173-198). Frankfurt a. M.: Peter Lang.

Lebedeva, M. (2012). Russkij jazyk s ulybkoj. Korotkie istorii, šutki, dialogi: posobie dlja izučajǔčich russkij kak inistrannyj. Moskau: Russkij jazyk.

Lejn, K. et al. (2006). Grosswörterbuch Deutsch - Russisch (13. Aufl.). Moskau: Russkij jazyk media. Lembens, A. \& Peschek, W. (2009). Was Fachdidaktiken sind und was sie wollen. Verfügbar unter: https://tinyurl.com/ydf3eu4w [02.12.2017].

Levina, G. (1997). Russkaja grammatika vanekdotach dlja načinajuščich. Sankt-Peterburg: Zlatoust. Lipps, Th. (1898). Komik und Humor, eine psychologisch-ästhetische Untersuchung. Hamburg, Leipzig: L. Voss.

Löschmann, M. (Hrsg.) (2015). Humor im Fremdsprachenunterricht. Frankfurt a. M.: Peter Lang.

Marfurt, B. (1977). Textsorte Witz. Möglichkeiten einer sprachwissenschaftlichen Textsorten-Bestimmung. Tübingen: Max Niemeyer.

Anka Bergmann, Olga Caspers \& Wolfgang Stadler (Hg.)

Didaktik der slawischen Sprachen - Beiträge zum 1. Arbeitskreis in Berlin (12.-14.9.2016)

(C) 2018 innsbruck university press, ISBN 978-3-903187-11-5, DOI 10.15203/3187-11-5 
Plessner, H. (1941). Lachen und Weinen. Eine Untersuchung nach den Grenzen menschlichen Verhaltens. Arnheim: v. Loghum Slaterus' Uitgeversmaatschappij.

Puškin, A. (1973). Skazka o rybake i rybke. Moskva: Detskaja literatura.

Preisendanz, W. (1970). Über den Witz. Konstanz: Universitätsverlag.

Raskin, V. (1985). Semantic Mechanisms of Humor. Dordrecht: D. Reidel.

Raskin, V. (Hrsg.) (2008). The Primer of Humor Research. Berlin: de Gruyter.

Shardakova, M. (2010). How to Be Funny in a Second Language: Pragmatics of L2 Humor. In R. Brecht, L. Verbitskaja, M. Lekic \& W. Rivers (Hrsg.), Mnemosynon. Studies on Language and Culture in the Russophone World: A Collection of Papers Presented to Da. E. Davidson by His Students and Colleagues (288-310). Moskau: Azbukovnik, Institut russkogo jazyka.

Shardakova, M. (2013). "I joke you don't": Second Language Humor and Intercultural Construction of Identity. In C. Kinginger (Hrsg.), Social and Cultural Aspects of Language Learning in Study Abroad (207-238). Amsterdam: John Benjamins.

Shardakova, M. (2016). American Learners' Comprehension of Russian Textual Humor. The Modern Language Journal, 100 (2), 466-483.

Šmeleva, E. \& Šmelev, A. (2002). Russkij anekdot: Tekst i rečevoj žanr. Moskau: Jazyki slavjanskoj kul'tury.

Stojić, A. \& Žagar-Šoštarić, P. (2015). Humor im interkulturellen Kontext. In M. Löschmann (Hrsg.), Humor im Fremdsprachenunterricht (277-295). Frankfurt a. M.: Peter Lang.

Ueda, Y. (2013). Textsorte Witz und Karikatur als Material zum Sprachlernen. Linguistische Ansätze zum Philosophieren mit Kindern. Münster: LiT Verlag.

Ulrich, W. (1980). Der Witz im Deutschunterricht. Braunschweig: Georg Westermann Verlag.

Vochmina, L. \& Osipova, I. (2011). Russkij klass. Učebnik russkogo jazyka. Načal'nyj uroven’. Moskau: Russkij jazyk.

Wellek, A. (1949). Zur Theorie und Phänomenologie des Witzes. Studium Generale, 2, 171-182.

Winkler, M. \& Goulding, Ch. (2005). Witz. In K. Barck et al. (Hrsg.), Ästhetische Grundbegriffe. Historisches Wörterbuch in 7 Bänden. (Band 6) (694-729). Stuttgart: Verlag J. B. Metzler. 